\title{
El caso de Lonchocarpus costaricensis (Leguminosae, Papilionoideae), una especie endémica de Costa Rica: un complejo taxonómico-nomenclatural, y una nueva especie
}

\author{
por \\ Nelson A. Zamora \\ Unidad Estratégica de Botánica, Instituto Nacional de Biodiversidad (INBio), Costa Rica. Apdo. 22-3100, Santo Domingo \\ nzamora@inbio.ac.cr
}

\section{Resumen}

Zamora, N.A. 2011. El caso de Lonchocarpus costaricensis (Leguminosae, Papilionoideae), una especie endémica de Costa Rica: un complejo taxonómico-nomenclatural, y una nueva especie. Anales Jard. Bot. Madrid 68(1): 7-14.

La mezcla de colecciones, la ausencia de la designación de un espécimen tipo y la fuerte similitud morfológica entre colecciones citadas en el protólogo de la descripción de Derris costaricensis generaron una situación taxonómica y nomenclatural confusa alrededor de la entidad Lonchocarpus costaricensis (Donn. Sm.) Pittier; un estudio taxonómico detallado del caso reveló que una nueva especie (Lonchocarpus felipei N. Zamora), aquí descrita, está implicada. Asimismo se restablece aquí la lectotipificación original de Lonchocarpus macrocarpus Benth.

Palabras clave: Taxonomía, nomenclatura, Leguminosae, Lonchocarpus, complejo de especies, lectotipificación, una nueva especie.

\section{Introducción}

En la descripción original de Derris costaricensis Donn. Sm., Bot. Gaz. 44: 110-111. 1907, el autor citó dos colecciones (síntipos), Tonduz 2880 (CR!, US!) y Tonduz 13993 (BM!, NY!, US!), que fueron utilizadas para elaborar el protólogo, sin dar indicación de la colección tipo. Más tarde, Pittier (1917) hizo la transferencia al género Lonchocarpus y designó Tonduz 13993 (fl y fr) como el lectótipo; en esta misma publicación, Pittier citó otras colecciones: Pittier 3654 (fr) (NY!, US!), y Tonduz 13528 (fl y fr) (F!, US!) y 13532 (fl y fr) (BM!, NY!, P!, US!).

Un estudio, aquí detallado, de todos los ejemplares

\begin{abstract}
Zamora, N.A. 2011. The case of Lonchocarpus costaricensis (Leguminosae, Papilionoideae), an endemic species of Costa Rica: a taxonomic-nomenclatural complex, and a new species. Anales Jard. Bot. Madrid 68(1): 7-14 (in Spanish).

Mixed collections, a lack of type specimen designation and strong morphological similarity between collections cited in the Derris costaricensis protologue has led to a very confused taxonomic and nomenclatural situation surrounding the species Lonchocarpus costaricensis (Donn. Sm.) Pittier; a detailed taxonomic study has revealed that a new species (Lonchocarpus felipei $\mathrm{N}$. Zamora) here described, is involved. Also, the original lectotipification of Lonchocarpus macrocarpus Benth., is re-establish it here.
\end{abstract}

Keywords: Taxonomy, nomenclature, Leguminosae, Lonchocarpus, species complex, lectotypification, a new species.

citados por Pittier (1917) y aquellos citados en el protólogo de Derris costaricensis determinó que Pittier 3654 (fr) y Tonduz 2880 (fr) corresponden a un taxon distinto. Este taxon posee una gran semejanza en su follaje y frutos con Lonchocarpus costaricensis (Donn. Sm.) Pittier. Debido y a partir de esta situación de mezcla de colecciones, el concepto de L. costaricensis se adoptó (p.e., Standley, 1937: 543) y se ha venido aplicando en forma equivocada. Además, ha favorecido tal confusión la condición del verdadero L. costaricensis, al ser una especie endémica de Costa Rica relativamente escasa o rara, mientras la aquí descrita (L. felipei N. Zamora) es más común y conocida ampliamente de Nicaragua y Costa Rica. 
Sousa (1990), consciente de esta situación (mezcla de colecciones) y aunque sin describirla y estudiarla en detalle, prefirió relectotipificar el nombre L. macrocarpus Benth., para acomodar la entidad aquí definida como distinta de L. costaricensis (Donn. Sm.) Pittier. Pero L. macrocarpus es un binomio heterogéneo compuesto de tres síntipos: Fendler 1861 (Venezuela), Orbigny 578 (Bolivia) y "New Spain, Herb Pavón" [lectótipo elegido por Sousa (1990)]. Sin embargo, ya previamente Pittier (1928), en ausencia de una designación de un tipo para L. macrocarpus, había seleccionado la colección Fendler 1861 (K!) como el lectótipo; designación con la que Sousa (1990) más tarde argumentó estar en desacuerdo con Pittier, señalando que fue una "escogencia mecánica", y propuso relectotipificar L. macrocarpus con el ejemplar Herb. Pavón s.n. (G!). El síntipo "Pavón" lleva anotado por Bentham una etiqueta "Nueva España Herb. Pavón” y marcada por Pavón "Classis 17 N 204 dubia N E. Securidaca?". La colección se compone de dos folíolos sueltos y frutos, cuya apariencia general (en especial por sus frutos) parece corresponder más bien a L. costaricensis.

La validez de la lectotipificación de L. macrocarpus hecha por Pittier había sido respaldada y aceptada por Tozzi (1989); además, a su vez este autor analizó con mayor detalle la naturaleza del concepto de L. macrocarpus, restringiéndolo al síntipo Fendler 1861 (K!), apoyando con esto la decisión de Pittier (1928). Vale mencionar también que, siguiendo este concepto del tipo [Fendler 1861], la mayoría del material recolectado de L. macrocarpus proviene del área geográfica del tipo (Venezuela). Para el síntipo Orbigny 578 (G!, P!), Tozzi (1989) no resolvió sobre su identificación; pero dicha colección fue identificada como Lonchocarpus hedyosmus Miq., por H.H. Poppendieck (HBG) en julio de 1993. También, según Tozzi (1989) y confirmado por este estudio, la colección Spruce 4597 (K!, G!, NY!, P!, W!; tipo, K!) de L. macrocarpus var. sericophyllus Benth., citada por Bentham (1860) en el protólogo de L. macrocarpus, corresponde a L. bedyosmus Miq.; identificación a su vez respaldada por H.H. Poppendieck (HBG), en julio de 1993. Finalmente, Tozzi (1989) señaló que L. hedyosmus y L. macrocarpus están altamente emparentadas. Pero en esta última las inflorescencias tienden a ser más cortas; el cáliz, denticulado, y los folíolos, persistentemente denso y sedoso-pubescentes en el envés, versus inflorescencias más alargadas, el cáliz truncado y folíolos glabrescentes o no denso sedoso-pubescentes en el envés en $L$. hedyosmus; también existen algunas diferencias a nivel de frutos.

Basándonos en la situación antes descrita, se concluye que la nueva lectotipificación establecida por
Sousa (1990) para L. macrocarpus no es válida, y considero más apropiado proponer un nombre nuevo para la especie históricamente identificada en forma equivocada como Lonchocarpus costaricensis o L. macrocarpus sensu Sousa (1990, 2001). Además, el restablecer la lectotipificación de L. macrocarpus hecha por Pittier estaría en mayor concordancia con la descripción que se provee en el protólogo original de L. macrocarpus, tanto con el tamaño del fruto, al cual el epíteto hace referencia, como en el número de folíolos (917) y área geográfica (Sudamérica) de dos de los síntipos citados [Fendler 1861(Venezuela) y Orbigny 578 (Bolivia)], excepto la colección Herb. Pavón s.n., cuya procedencia específica sigue siendo aún dudosa o sin resolver (véase McVaugh, 2000: 322). Por lo que se conserva aquí el lectótipo seleccionado por Pittier (1928): Lonchocarpus macrocarpus Benth., J. Linn. Soc., Bot. 4 (Suppl.): 91. 1860. Tipo: "Fendler 1861 (Venezuela)” (lectótipo, K!, aquí redesignado). El restablecer la lectotipificación de Pittier (1928) significa a su vez oficializar aquí, por primera vez, que la especie Lonchocarpus margaritensis Pittier es sinónimo de Lonchocarpus macrocarpus Benth (véase Tozzi, 1989; Tozzi \& Silva, 2007).

La especie aquí descrita es ampliamente conocida (según abundante material de herbario citado y examinado) y estudiada por ecólogos y biólogos en la Provincia de Guanacaste (Costa Rica) desde mediados de los años 1960, mientras la verdadera (endémica, algo rara y más localizada) Lonchocarpus costaricensis permanece más o menos oculta o aislada en algunas colinas del Área de Conservación Guanacaste (especialmente Sector Murciélago) y algunos cerros de formaciones calcáreas en la Península de Nicoya.

\section{Lonchocarpus felipei N. Zamora, sp. nov.}

Tipo: Costa Rica. Puntarenas: Cantón de Puntarenas, ridges between Río Guacimal and Río Lagarto on road from Inter American Highway to Monteverde, $10^{\circ} 16^{\prime} \mathrm{N}, 84^{\circ} 50^{\prime} \mathrm{W}, 800-1000$ m, 20 April 1991 (fl), Haber E Zuchowski 10656 (holótipo, INB; isótipos, CR, K, MEXU, MO, PMA,). Figs. 1, 2.

Lonchocarpus costaricensis similis, sed floribus minoribus [usque ad 9-10(12) mm, non 15-18(20) mm], vinaceis vel atropurpureis (non roseis) recedit; a Lonchocarpus retifer foliolis paucioribus, fructibus latioribus differt.

Árbol de 7-14 m de alto, ramitas conspicuamente lenticeladas, denso a esparcido ferrugíneo pubescentes hacia el ápice o partes jóvenes; estípulas deciduas, no vistas. Hojas imparipinnado-compuestas, con 5-7(9) folíolos; pecíolo de 5,5-11 cm de largo; ra- 


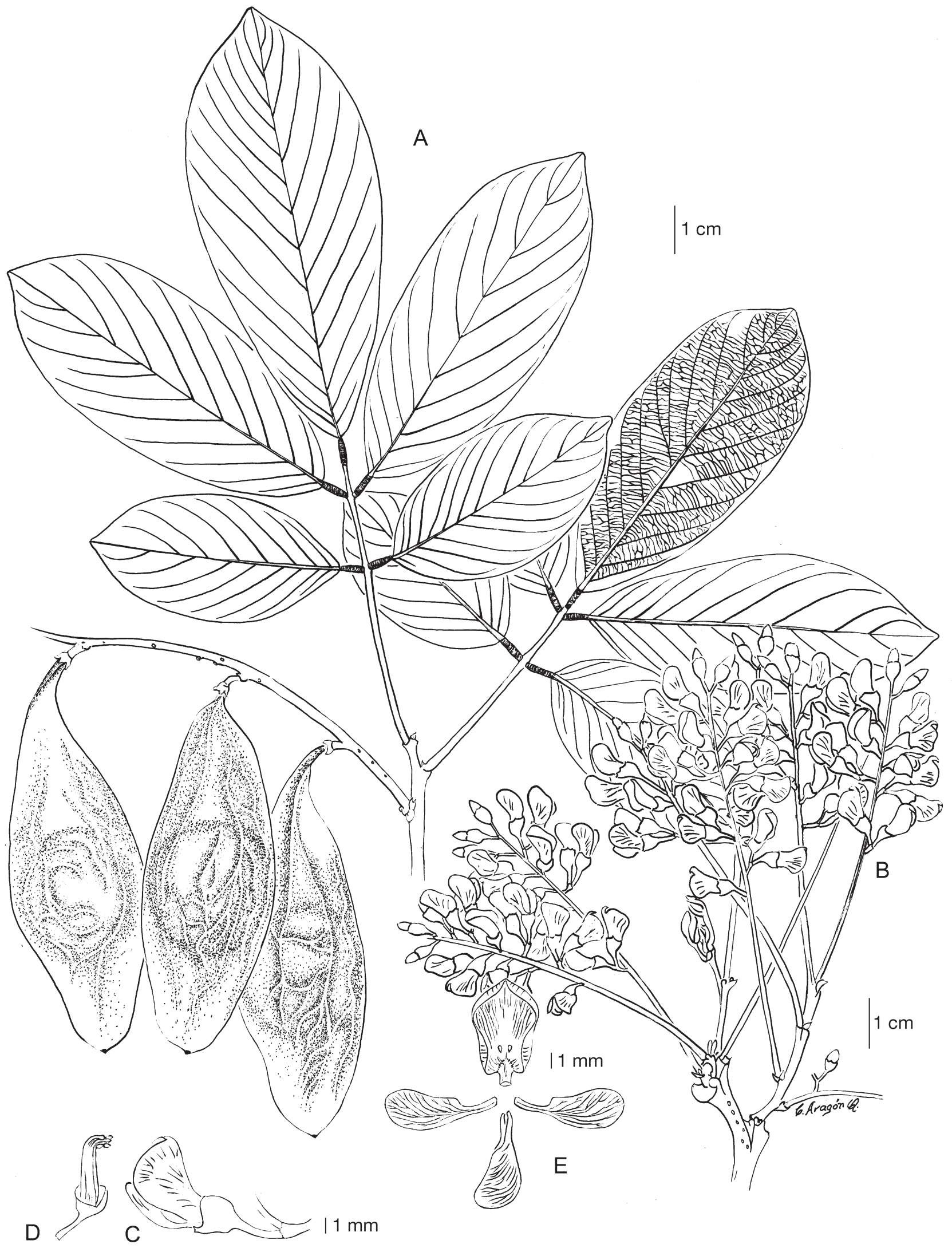

Fig. 1. Lonchocarpus felipei: A, rama con frutos (Zamora 2281, INB); B, rama con inflorescencias; C, flor; D, estambres, E, pétalos (Haber \& Zuchowski 10656, INB). Ilustración de Claudia Aragón. 
quis de 4-10 cm de largo, levemente caniculado, glabrescente a ferrugíneo pubescente, peciolulos $5-9 \mathrm{~mm}$ de largo; folíolos distales (6,5)9-21 × 3,6-10,2 cm, oblongos a obovados, mediales (5)9-14,5 × (3)3,9-7,5 $\mathrm{cm}$, oblongos a obovados, basales de (4)6-11 $\times(2,4)$ 3,6-7,7 cm, ovados a oblongos o suborbiculares, ápice redondeado, base aguda, obtusa a levemente asimétrica, glabros cuando adultos o esparcido pilosos cuando jóvenes en el haz, glabrescentes a ferrugíneo pilosos en el envés, nervios secundarios 9-17 por lado, venación terciaria conspicuamente reticulada. Inflorescencias racemosas, axilares, $2-16 \mathrm{~cm}$ de largo, eje principal glabrescente a diminuto estrigoso o esparcido seríceo, pedúnculos o ejes laterales secundarios de 1-5 mm de largo; brácteas deciduas, no vistas, bractéolas 0,5-0,6 mm, escuamiformes, decíduas. Flores
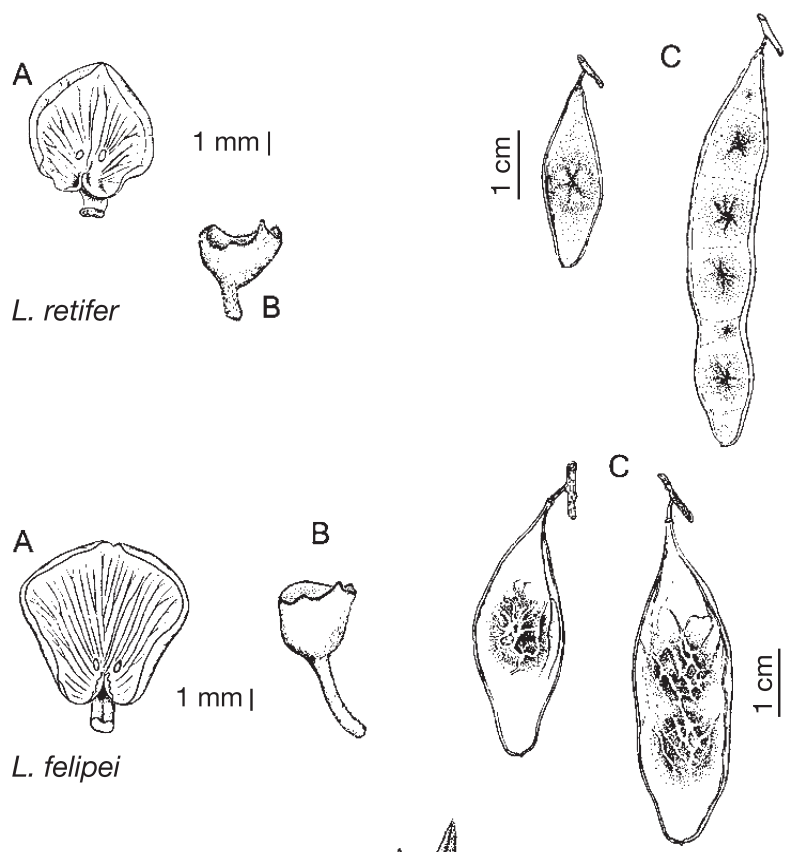

L. felipei

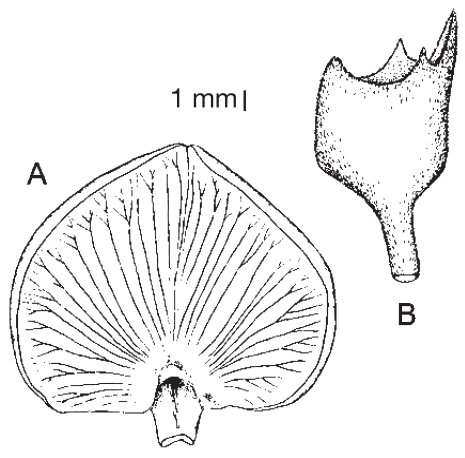

L. costaricensis

Fig. 2. A, estandarte; B, cáliz; C, frutos de: Lonchocarpus retifer (flores, Espinoza \& al. 1472, INB; frutos, Morales 5559, INB), L. felipei (flores, Haber \& Zuchowski 10656, INB; frutos, Zamora 2281, INB) y L. costaricensis (flores, Zamora \& al. 2243, INB; frutos, González \& Garita 3052, INB). Ilustración de Claudia Aragón. de color marrón a púrpura oscuro, 9-10(12) mm de largo; pedicelo 3,5-4 mm de largo; cáliz (1,5)2-3 mm de largo, cupuliforme, diminuto seríceo, truncado o ligeramente ondulado-denticulado; pétalos 5 , estandarte 8-11(12) × 9-10 mm, suborbicular y cóncavo, con los márgenes levemente involutos, ligeramente recurvado, nervado, seríceo por fuera y glabro por dentro, con una mácula verde basal y punteado cerca de la base en la cara interna, emarginado en ápice y auriculado en la base, la uña ca. $2 \mathrm{~mm}$ de largo; alas 5-7 $\times$ 2,5-3 3,5$) \mathrm{mm}$, oblongo-oblicuas, redondeadas en el ápice y levemente auriculadas en la base, la uña ca. $3 \mathrm{~mm}$; quilla 5-6 × 3-3,2 mm, oblongo-oblicua, unida distalmente, diminuto serícea por fuera, uña ca. 2,8-3 mm; tubo estaminal 6-6,5 mm, glabro; pistilo linear, 6-7,5 mm, denso seríceo, óvulos 1(2); estilo fuertemente recurvado; estigma inconspicuo; anteras basifijas. Frutos legumbres, 7-12(16) × 3-5 cm, elípticos a ovado-elípticos u obovado-elípticos a veces falcadoelípticos, redondeados en el ápice y obtusos a atenuados en la base, coriáceos, ambos márgenes afilados, pardo-amarillentos, glabros o glabrescentes cuando adultos y pardo-amarillento sedoso pubescentes cuando jóvenes, con verrugas evidentes a la altura de las semillas; semillas 1 ó 2, 1,5-1,6 ×0,8 cm, aplanadas, oblongo-reniformes, pardo-rojizas cuando secas.

A pesar de la larga confusión en la que L. costaricensis estuvo inmersa, ésta difiere de L. felipei de manera notable, como se resume en el siguiente cuadro (Tabla 1).

Ambas especies poseen hojas con 5-7 folíolos y éstos son muy semejantes en su apariencia general, por lo que es algo difícil diferenciarlas en forma vegetativa, aunque las ramitas y hojas (raquis y envés de los folíolos) de L. costaricensis poseen una pubescencia tomentosa pardo-rojiza o ferrugíneo-oscura; mientras, en L. felipei la pubescencia es más amarillenta a pardo-amarillenta.

A nivel de flores (Fig. 2), tamaño y color, y en cierto grado los frutos, la mayor afinidad de $L$. felipei es más bien con la especie L. retifer Standl. \& L.O. Williams, pero en ésta última sus flores son relativamente más pequeñas $(5-6 \mathrm{~mm})$, las hojas normalmente tienen más folíolos (comúnmente 9) y sus frutos son más angostos $(2-2,6(2,9) \mathrm{cm})$. Además, L. retifer se da en climas más húmedos y su floración ocurre cuando el árbol tiene hojas.

Hábitat, distribución, conservación y ecología: Lonchocarpus felipei es propio de bosques secos a húmedos, a lo largo de la costa pacífica de Nicaragua (Chontales) hasta Costa Rica (Valle Central); es de frecuente a común en vegetación caducifolia o semicaducifolia, de preferencia en terreno sedimentario, 
Tabla 1. Principales caracteres diferenciadores entre Lonchocarpus felipei y L. costaricensis. Medidas de flores para $L$. felipei fueron tomadas de Haber \& Zuchowski 10656 (fI) (INB!) y para L. costaricensis tomadas de Zamora \& al. 2243 (fl) (INB!).

\begin{tabular}{|c|c|c|}
\hline Carácter & L. felipei & L. costaricensis \\
\hline Flores, color & marrón o púrpura oscuro & rosadas \\
\hline Flores, tamaño $(\mathrm{mm})$ & $9-10(12)$ & $15-18(20)$ \\
\hline Estandarte, mácula & presente, verde & ausente o no diferenciada \\
\hline Cáliz, tamaño (mm) & $(1,5) 2-3$ & $4-5$ \\
\hline Cáliz, forma & cupuliforme & campanulado \\
\hline Cáliz, margen & truncado a ondulado-denticulado & dentado, el vexilar 2-3 mm \\
\hline Bractéolas, forma & escuamiformes & filiformes \\
\hline Bractéolas, tamaño (mm) & 0,5-0,6 mm, deciduas & 5-7 mm, persistentes \\
\hline Inflorescencia, pubescencia & diminuto estrigosa o serícea esparcida & denso ferrugíneo tomentosa \\
\hline Frutos adultos & $\begin{array}{l}\text { verdoso-amarillentos o pardo-amarillentos, } \\
\text { glabros, glabrescentes a tomentosos, } \\
\text { con verrugas a la altura de las semillas }\end{array}$ & $\begin{array}{l}\text { pardo-rojizos o ferrugíneo-oscuros, } \\
\text { denso tomentosos, } \\
\text { lisos a la altura de las semillas }\end{array}$ \\
\hline Hábitat & $\begin{array}{l}\text { bosque seco a húmedo, } \\
\text { prefiere terreno sedimentario }\end{array}$ & $\begin{array}{l}\text { bosque seco, } \\
\text { prefiere terreno cálcareo }\end{array}$ \\
\hline Distribución & Nicaragua-Costa Rica & Endémica, Costa Rica \\
\hline
\end{tabular}

desde el nivel del mar hasta los 800(1000) m. Al menos en Costa Rica la especie se encuentra protegida y poblaciones importantes se encuentran en el Área de Conservación Guanacaste (ACG: Sector Santa Rosa, Sector Santa Elena, Sector Murciélago, Sector Pocosol), Parque Nacional Palo Verde, Refugio de Vida Silvestre Macacona-Esparza y Zona Protectora El Rodeo-San José. Su floración ocurre de febrero a abril, siendo máxima cuando el árbol está completamente caducifolio. Es interesante añadir que he observado el fenómeno del albinismo en un individuo en el Sector Santa Rosa, ACG; fenómeno también visto en Lonchocarpus cultratus (Vell.) A.M.G. Azevedo \& H.C. Lima (A.M.G. Azevedo Tozzi, com. pers., 2010). Sus frutos se han observado la mayor parte del año, aunque permanecen en el árbol durante toda la estación lluviosa y maduran y caen en el primer mes del verano siguiente; cada individuo reproductivo florece un año y tiene frutos hasta el siguiente, pero no sincronizadamente entre árboles (D.H. Janzen, com. pers., 2010). Ensayos de reproducción y propagación han dado buenos resultados, con importantes porcentajes de germinación y desarrollos en vivero y campo.

Es importante mencionar que toda la literatura ecológica, química y molecular (p.ej., Chapman, 1989; Evans \& al., 1985; Fellows \& al., 1979; Janzen, 1980, 1982, 1983, 1986; Janzen \& Liesner, 1980; Janzen \& al., 1990; Navarro \& al., 2005; Waterman \& Mahmoud, 1985) generada para la especie (o donde se cita el nombre) Lonchocarpus costaricensis corresponde más bien a la especie aquí descrita. A menudo su follaje se encuentra altamente infestado de agallas, provocadas por insectos hemípteros del género $E$ - phalerus, situación frecuente que padecen varias especies de Lonchocarpus (Hollis \& Martin, 1997).

Etimología: Dedico esta especie en honor a su Alteza Real el Príncipe Don Felipe de Borbón, por su apoyo al estudio de la biodiversidad de Costa Rica.

\section{Colecciones examinadas}

COSTA RICA. Guanacaste: Cantón de La Cruz, P.N. Guanacaste, Cordillera de Guanacaste, Camino a la Estación Maritza, a orillas del Río Espavelar, 1058'10”'N, 85³3'35'W, 300 m, 25-V1995 (fr), Zamora 2290 (MO); Bahía Salinas a Santa Cecilia, camino a la Estación Maritza, alrededores de la Quebrada Espavelar,

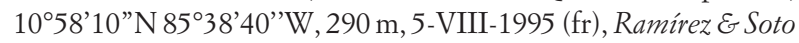
392 (INB); Cuajiniquil, camino entre Cuajiniquil y Junquillal, 1057'20”N, 8542'00”W, 0 m, 8-IX-1995 (fr), J. Sánchez 534 (CR). Cantón de Liberia, Parque Nacional Santa Rosa, Llano Jicaral, hacia la playa, 280 m, 25-I-1983 (fr), Sousa \& al. 12688 (MO); Parque Nacional Santa Rosa, Llano Jicaral, hacia la playa, 280 m, 25-I-1983 (fr), Sousa 12688 (CR); Parque Nacional Santa Rosa, 10 m, 4-XII-1985 (fr), Zamora E al 1152 (CR, MO); Parque Nacional Santa Rosa, bosque seco, 317 m, 3-IV-1976, Tinney 236 (CR); Parque Nacional Santa Rosa, bosque secundario, 317 m, 3-I1976, Chazdon 210 (CR); Parque Nacional Santa Rosa, entrada al mirador Valle Naranjo, $10^{\circ} 48^{\prime} 00^{\prime} \mathrm{N}, 85^{\circ} 38^{\prime} 37^{\prime \prime} \mathrm{W}, 200 \mathrm{~m}, 11-\mathrm{IV}$ 2000 (str), Acosta E al. 847 (MO); Liberia, Parque Nacional Santa Rosa, alrededor de la entrada a Nancite y playa Naranjo, $10^{\circ} 48^{\prime} 30^{\prime \prime} \mathrm{N}, 85^{\circ} 40^{\prime} 55^{\prime \prime} \mathrm{W}, 10 \mathrm{~m}, 26-\mathrm{IV}-2000$ (fr), Acosta E al. 931 (INB, MO); faja costeña del golfo de Papagayo, Hacienda Horizontes, 1042'25”N, 8334'30'W, 130 m, 1-III-1995 (st), Zamora 2241 (MO). Cantón de Carrillo, Península de Nicoya, Sardinal Nuevo Colón, 2-3 km después del cruce a Zapotal, cerro Judas, camino a playa Guacamaya, $10^{\circ} 29^{\prime} 45^{\prime} \mathrm{N}, 85^{\circ} 44^{\prime} 05^{\prime \prime} \mathrm{W}, 29 \mathrm{~m}, 24-\mathrm{V}$ 1995 (fr), Zamora E al. 2285 (INB); faja costeña del golfo de Papagayo, Sardinal alrededores playa Monte del Barco, 10³6’30”N, 85³8'20"W, 20 m, 3-II-1996 (fr), Jiménez E al. 2065 (INB); bahía El Coco, bahía Playa Hermosa, and Sardinal, 10³2’00”N, 8540'00"W, 0-150 m, 10-XI-1975 (fr), Burger E Baker 9933 (CR); 

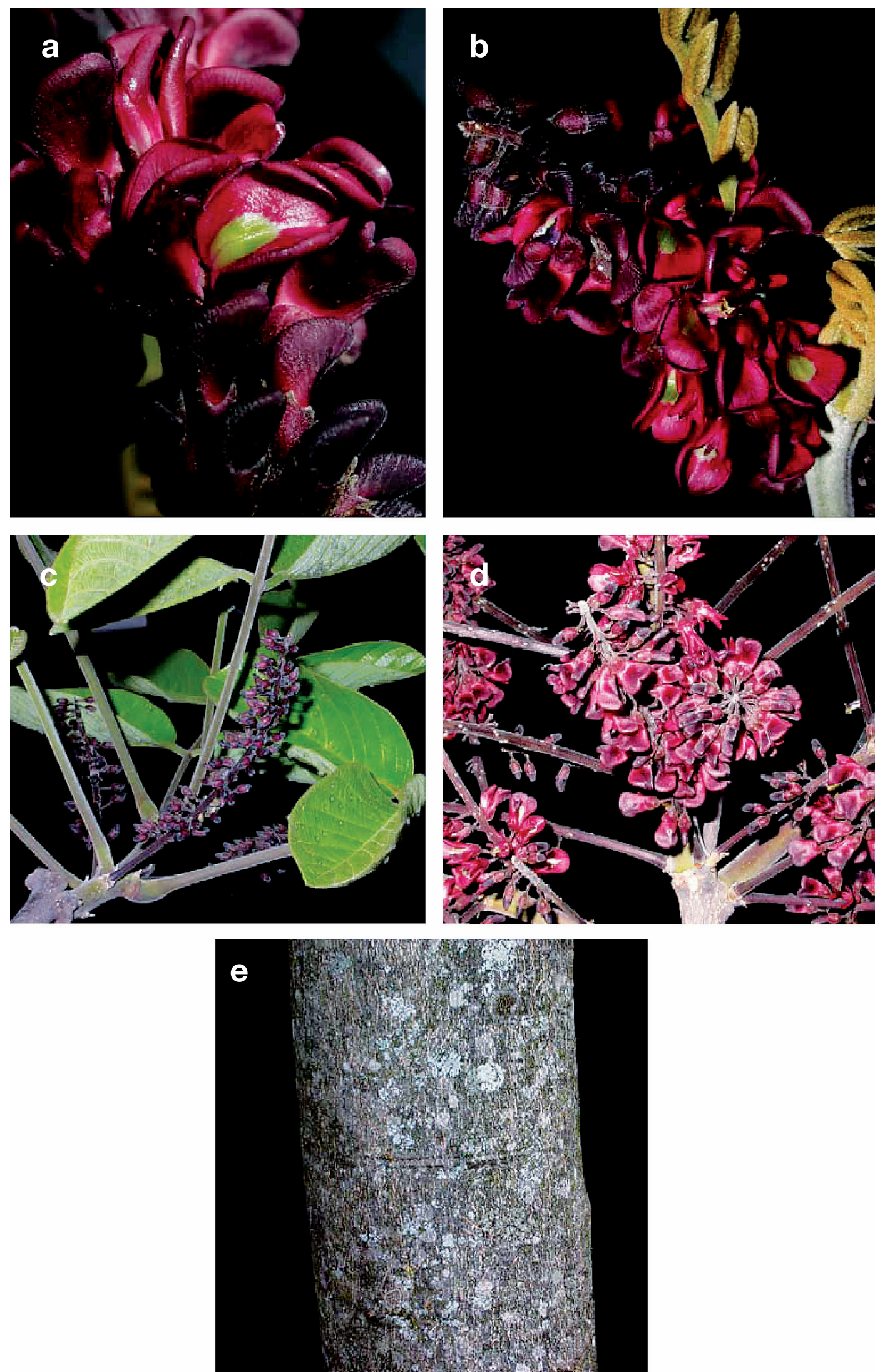

Fig. 3. Lonchocarpus felipei: a, b, flores maduras, mostrando el color vivo, el estandarte reflexo y mácula verde; c, rama con inflorescencias inmaduras, con hojas; d, rama en máxima floración, sin hojas; e, tallo, con la corteza lisa. Fotografías de D. Solano (Zamora \& Solano 4913, INB). 
cerro el Hacha, 28-VII-1986 (fr), Chacón E Chacón 2038 (CR, $\mathrm{MO}$; faja costeña del golfo de Papagayo, camino entre Nuevo Colón y Zapotal, cerca del cerro Matapalo. 10³0’30”N 8546'20"W, 300 m, 4-VIII-1995 (fr), Ramírez \& Soto 384 (INB), 386 (INB). Cantón Santa Cruz, 4 km E. Santa Cruz, 130 m, XII1973, Solomon 667, (CR); Cañas, La Pacífica, 24-VIII-1990 (fr), Jiménez 54 (CR). Cantón de Bagaces, Parque Nacional Palo Verde, valle del Tempisque, cerro Jocote, sector Carreta, $10^{\circ} 22^{\prime} 45^{\prime} \mathrm{N}$, 85¹9'15'W, 0-100 m, 11-VI-1993 (fr), Chavarría 822 (INB, CR); Parque Nacional Palo Verde, Estación Catalina, sendero Botija, saliendo al Cenicero, $10^{\circ} 21^{\prime} \mathrm{N}, 85^{\circ} 16^{\prime} \mathrm{W}, 10-20 \mathrm{~m}$, 9-XII-1991 (fr), Chavarría 450 (INB, CR, MO); R.B. Lomas Barbudal, valle del Tempisque; Lomas Barbudal, Marañonal, Oja de Agua y Agua Fría, $10^{\circ} 26^{\prime} 25^{\prime} \mathrm{N}, 85^{\circ} 19^{\prime} 05^{\prime} \mathrm{W}, 100-200 \mathrm{~m}, 2-\mathrm{V}-1993$ (fr), Chavarría 797 (INB); Cuenca del Temspisque, Hacienda Monteverde, 10³3'00'N, 85¹8'20'W, 100-300 m, 9-VI-1996 (fl), Ronchi E Frankie 830 (INB); Cuenca del Tempisque, $3,5 \mathrm{~km}$ del cruce al Parque, sobre la carretera Interamericana, $10^{\circ} 29^{\prime} 50^{\prime \prime} \mathrm{N}$, $85^{\circ} 15^{\prime} 40^{\prime \prime} \mathrm{W}, 70$ m, 23-XI-2000 (fr), Acosta E al. 3009 (INB); Refugio de Vida Silvestre-Palo Verde, sin fecha (fl), Ramírez 220 (CR); finca La Pacífica, 2 miles N of Cañas along Pan-Am Hwy., gallery forest between río Corobicí and irrigation ditch, 5-VI-1971 (fr), Gentry 810 (CR, MO); Lomas Barbudal, Bagaces 100 m, 10V-1984 (fr), Gómez 23012 (CR, MO); road from Bagaces to Aguas Claras about $5 \mathrm{~km}$ North of Bagaces, 150-200 m, 7-VII-1976 (fr), Utley 5305 (MO, CR). Cantón de Cañas, Hacienda La Pacífica near Cañas, 14-VIII-1986 (fr), Seigler 12774 (MO), 12401A (MO); Comelco Ranch, Bagaces (MO), remnant forest along stream, $2 \mathrm{~km}$

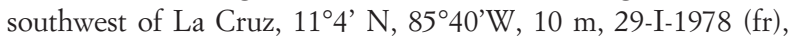
Liesner 4635 (CR, MO); seasonal swamp, dry now, OTS Área A3 site a Comelco, 31 March 1972 (fl), Stone E Opler 3157 (MO); Comelco Ranch, $10^{\circ} 20-35^{\prime} \mathrm{N}, 85^{\circ} 18-25^{\prime} \mathrm{W}, \mathrm{V}-1970$ (fr), Hartshorn 910 (MO); Palo Verde, 4-VI-1969 (fr): valle del Tempisque, cerca del río Lajas, 174-175, $600 \mathrm{~m}$ antes de la Escuela de Buenos Aires, $10^{\circ} 19^{\prime} 40^{\prime \prime N}, 5^{\circ} 03^{\prime} 10^{\prime \prime} \mathrm{W}, 120$ m, 3-VIII-1995 (fr), Ramírez \& Soto 383 (INB). Cantón de Abangares, valle del Tempisque, Abangares, orillas de la carretera Interamericana ruta 1, 173-74, $1 \mathrm{~km}$ después del río Lajas, $10^{\circ} 19^{\prime} 10^{\prime \prime} \mathrm{N}, 85^{\circ} 02$ '50"'W, 100 m, 20-IV1991 (fr), Zamora 2281 (MO); valle del Tempisque, orillas de la carretera Interamericana, ruta 1, km 173-174, entre Lourdes y río La-

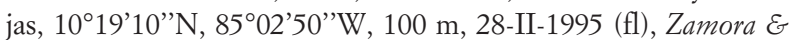
Mora 2239 (INB). Puntarenas: Cantón de Puntarenas, Hacienda Santa Marta, Cascajal, along ditch near Cascajal Station $(25 \mathrm{~km}$ ESE of Puntarenas), 2-VI-1949 (fr), Holm E Iltis 221 (BM, MO, $\mathrm{U})$; ridges between río Guacimal and río Lagarto on road from Inter American Highway to Monteverde, $10^{\circ} 16^{\prime} \mathrm{N}, 84^{\circ} 50^{\prime} \mathrm{W}, 800$ 1000 m, 20-IV-1991 (fls), Haber E Zuchowski 10653 (INB, CR), 10656 (INB, CR); Monteverde, valle del río Guacimal, Lindora, vertiente pacífica, $10^{\circ} 18^{\prime} \mathrm{N}, 84^{\circ} 50^{\prime} \mathrm{W}, 1000 \mathrm{~m}, 17-\mathrm{VI}-1998$ (fr), BeIlo 402 (CR, MO); Monteverde, road from Santa Elena to village of San Luis and Lagarto, Pacific slope, moist forest, $10^{\circ} 16^{\prime} \mathrm{N}$, 8450'W, 750-900 m, 10-VII-1990 (fr), Haber 9987 (CR, INB, MO); Santa Elena to Coyolar de Guacimal, Pacific Slope, roadside and remnant forest patches of dry forest, $10^{\circ} 15^{\prime} \mathrm{N}, 84^{\circ} 51^{\prime} \mathrm{W}, 250$ 900 m, 16-VII-1988 (fr), Hammel 17111 (CR). R.N.A. Cabo Blanco, estación Cabo Blanco, bosque primario y secundario, $9^{\circ} 35^{\prime} \mathrm{N}$, 8506'W, 20-100 m, 4-XI-1991 (fr), Chavarría 295 (INB, CR, MO); R.N.A. Cabo Blanco, Camaronal, sendero el Barco, 9³4'42'N, 8508'10'W, 0 m, 25-VI-2001 (fr), Chavarría E al. 2183 (INB); isla San Lucas, golfo de Nicoya, 957'N, 8454'W, 0-400 m, 20-X-1984 (fr), Grayum E al. 4236 (MO); R.N.A. Cabo Blanco, península de Nicoya, sendero a El Atracadero de San Miguel, 9³5'00'N ,8507’00”W, 1-300 m, 17-XII-1993 (fr), Fernán- dez E al. 1300 (INB); golfo de Nicoya, isla San Lucas, entre playa Cocos y playa Bellavista, 957'18'N, 8454'11''W, 20 m, 17-III2005 (fl), Soto E González 539 (INB); Punta Morales, 1-3 m, 19VII-1984 (fr), Gómez-Laurito 10071 (CR). San José: Cantón de San José, Villa Colón, 800 m, 19-I-1972 (fr), Caffrey 86 (CR); vicinity of Villa Colón, 15-II-1965 (fl), Godfrey 66479 (MO). Cantón de Mora, Zona Protectora El Rodeo, camino que desciende de la Universidad a La Paz hacia el río Jaris, pequeños bosquetes a orilla del camino junto a potreros y cultivos, $9^{\circ} 54^{\prime} 00^{\prime} \mathrm{N}, 84^{\circ} 16^{\prime} 00^{\prime} \mathrm{W}$, 500-1000 m, 8-VII-1996 (fr), Cascante 1040 (CR); Z.P. El Rodeo, valle del Tárcoles, bajo morales, 955'00”N $84^{\circ} 16^{\prime} 00^{\prime}$ 'W, 8001000 m, 08-VII-1995 (st), Jiménez E Ramírez 1886 (MO). Cantón de Acosta, valle del Candelaria, cuenca del río Candelaria, parches remanentes y potreros cerca del puente, 9 $9^{\circ} 46^{\prime} 50^{\prime \prime} \mathrm{N}, 84^{\circ} 11^{\prime} 43^{\prime \prime} \mathrm{W}$, 700 m, 19-XI-1994 (fr), Morales 3155 (INB, CR, MO); Cantón de Orotina, N outskirts of Orotina, 9 ${ }^{\circ} 55^{\prime} \mathrm{N}, 84^{\circ} 32^{\prime} \mathrm{W}, 200 \mathrm{~m}$, 6-IV1983 (fr), Judziewicz 4559 (CR).

NICARAGUA. Chontales: $0,9 \mathrm{~km} \mathrm{NE}$ of Hwy 7 on road to Comalapa, ca. $12^{\circ} 10^{\prime} \mathrm{N}, 85^{\circ} 33^{\prime} \mathrm{W}, 160 \mathrm{~m}, 12-\mathrm{VI}-1982$ (fr), Stevens $\mathcal{E}$ al. 21570 (MO). Granada: $\mathrm{km} \mathrm{75,} \mathrm{carretera} \mathrm{Sur,} 8 \mathrm{~km}$ de Nandaime, Llanos el Dorado, $11^{\circ} 41^{\prime} \mathrm{N}, 86^{\circ} 00^{\prime} \mathrm{W}, 70$ m, 25-I-1984 (fr), Moreno E Stevens 22855 (MO); camino de Casa Tejas, $1,2 \mathrm{~km}$ antes de la finca San José del Mombacho, $11^{\circ} 46^{\prime} \mathrm{N}, 85^{\circ} 54$ 'W, 40-60 m, 21-VI-1982 (fr), Moreno 16644 (MO); camino a Charco Muerto, $3 \mathrm{~km}$ al E de Casa de Tejas, sobre el camino, 100-200 m, 5-VI1980 (fr), Araquistain \& Moreno 2855 (MO). Managua: carretera a Montelimar, comarca Aduana, al N del río Aduana, 80-100 m, 21VII-1980 (fr), Guzmán E al. 410 (MO). Rivas: along road SE from San Juan del Sur, 3-4 km NW of río La Flor, playa El Coco, quebrada El Coco, $11^{\circ} 09^{\prime} \mathrm{N}, 85^{\circ} 47^{\prime} \mathrm{W}, 0-95$ m, 17-XII-1977 (fr), Stevens $5492(\mathrm{MO})$.

\section{Agradecimientos}

Deseo expresar un profundo agradecimiento a mis colegas Claudia Aragón, por la preparación de las ilustraciones, y Henk van der Werff, por su asistencia en la elaboración de la diagnosis en latín. A Michael H. Grayum, A.M.G.A. Tozzi, Lourdes Rico, D.H. Janzen y los revisores de esta revista, por sus valiosos aportes, comentarios y sugerencias en una versión preliminar de este artículo. Además, a todos los herbarios citados, en especial aquellos que albergan colecciones históricas, por facilitarme acceso y ayuda para el estudio de las mismas.

Esta investigación fue posible gracias al convenio de cooperación entre el Ministerio de Ambiente, Energía y Telecomunicaciones (MINAET) y el Instituto Nacional de Biodiversidad (INBio), al apoyo económico de particulares y a la red de especialistas taxónomos que contribuyen a completar el Inventario Nacional de Biodiversidad en Costa Rica.

\section{Referencias bibliográficas}

Bentham, G. 1860. Synopsis of Dalbergieae, a tribe of Leguminosae. Journal of Linnean Society Botany 4 (Suppl.): 1-28.

Chapman, C.A. 1989. Primate Seed Dispersal: The Fate of Dispersed Seeds. Biotropica 21(2): 148-154.

Evans, S. , Fellows, L.E., Janzen D.H., Chambers, J., \& Hider, R.C. 1985. Erythro-gamma-hydroxyhomo-L-arginine: an amino acid from seed of Lonchocarpus costaricensis, and its preferential interaction with borate. Photochemistry 24: 1289. 1292. 
Fellows, L.E., Bell, E.A., Lee, T.S. \& Janzen, D.H. 1979. Tetrahydrolathyrine; a new amino acid from seeds of Lonchocarpus costaricensis. Phytochemistry 18: 1333-1335.

Hollis, D. \& J.H. Martin. 1997. Jumping plantlice (Insecta: Hemiptera) attacking Lonchocarpus species (Leguminosae), including "Black Cabbage Bark", in Belize. Journal of Natural History 31: 237-267.

Janzen, D.H. 1980. Specificity of seed-attacking beetles in a Costa Rican deciduous forest. Journal of Ecology 68: 929-952.

Janzen, D.H. 1982. Weight of seeds in 1-3 seeded fruits of Lonchocarpus costaricensis (Leguminosae), a Costa Rican winddispersed tree. Brenesia 19/20:363-368.

Janzen, D.H. 1983. Costa Rican Natural History. University of Chicago Press, Chicago. 816 pp.

Janzen, D.H. 1986. Mice, big mammals, and seeds: it matters who defecates what where. In: Estrada, A. \& Fleming, T.H. (eds), Frugivores and seed dispersal. Pp. 251-271. Dr. W. Junk Publishers, Dordrecht.

Janzen, D.H. \& Liesner, R. 1980. Annotated check-list of plants of lowland Guanacaste Province, Costa Rica, exclusive of grasses and non-vascular cryptogams. Brenesia 18: 15-90.

Janzen, D.H., Fellows, L.E. \& Waterman, P.G. 1990. What protects Lonchocarpus (Leguminosae) seeds in a Costa Rican dry forest? Biotropica 22: 272-285.

McVaugh, R. 2000. Botanical Results of the Sessé E Mociño Expedition (1787-1803). VII. A Guide to Relevant Scientific Names of Plants. Hunt Institute for Botanical Documentation. Carnegie Mellon University, Pittsburgh.

Navarro, C., S. Cavers, N. Colpaert, G. Hernández, P. Breyne \& A.J. Lowe. 2005. Chloroplast and Total Genomic Diversity in the Endemic Costa Rican Tree Lonchocarpus costaricensis (J.
Donn. Smith) Pittier (Papilionaceae). Silvae Geniticae 54(6): 293-300.

Pittier, H. 1917. The Middle American species of Lonchocarpus. Contributions from the United States National Herbarium 20: 37-93.

Pittier, H. 1928. Contribuciones a la dendrología de Venezuela. Árboles y arbustos del orden de las Leguminosas. III-Papilionáceas. Trab. Mus. Com. Venezuela (Bol. Minist. R. R. E. E. n. $\left.{ }^{\circ} 4-7\right)$ 4: 179-259.

Sousa, M. 1990. Adiciones a las Papilionadas de la flora de Nicaragua y una nueva combinación para Oaxaca, México. Annals of the Missouri Botanical Garden 77: 573-577.

Sousa, M. 2001. Lonchocarpus. In: Stevens, W.D., Ulloa, C., Pool, A. \& Montiel, O. (eds.), Flora de Nicaragua. Monographs in Systematic Botany from the Missouri Botanical Garden 85(2): 1-2666. Pp. 1017-1028.

Standley. P. 1937-1938. Flora of Costa Rica. Field Museum of Natural History. Botanical Series 18: 1-1571.

Tozzi, A.M.G.A. 1989. Estudos taxonômicos dos gêneros Lonchocarpus Kunth e Deguelia Aubl. no Brasil. Tesis doctoral. Universidade Estadual Campinas, Campinas.

Tozzi, A.M.G.A. \& Silva, M.J. 2007. Sinonimizações em Lonchocarpus Kunth (Leguminosae-Papilionoideae-Millettieae). Rodriguesia 58(2): 275-282.

Waterman, P.G. \& Mahmoud, E.N. 1985. Flavonoids from the seeds of Lonchocarpus costaricensis. Photochemistry 24(3): 571-574.

Editor asociado: L. Rico Recibido: 17-III-2010 Aceptado: 1-XI-2010 